\title{
From Gut to Brain: Alteration in Inflammation Markers in the Brain of Dextran Sodium Sulfate-induced Colitis Model Mice
}

\author{
Jongho Do ${ }^{1}$, Jungmin Woo ${ }^{1,2}$ \\ ${ }^{1}$ Department of Psychiatry, Kyungpook National University Hospital, ${ }^{2}$ Depatment of Psychiatry, School of Medicine, Kyungpook National \\ University, Daegu, Korea
}

\begin{abstract}
Objective: Neuropsychiatric manifestations like depression and cognitive dysfunction commonly occur in inflammatory bowel disease (IBD). In the context of the brain-gut axis model, colitis can lead to alteration of brain function in a bottom-up manner. Here, the changes in the response of the hypothalamic-pituitary-adrenal axis and inflammationrelated markers in the brain in colitis were studied.

Methods: Dextran sodium sulfate (DSS) was used to generate a mouse model of colitis. Mice were treated with DSS for 3 or 7 days and sacrificed. We analyzed the gene expression of brain-derived neurotrophic factor (BDNF), cyclooxygenase 2 (COX-2), and glial fibrillary acidic protein (GFAP), and the expression of GFAP, in the hippocampus, hypothalamus, and amygdala. Additionally, the levels of C-reactive protein (CRP) and serum cortisol/corticosterone were measured.

Results: Alteration of inflammatory-related markers varied depending on the brain region and exposure time. In the hippocampus, COX-2 mRNA, GFAP mRNA, and GFAP expression were upregulated during exposure to DSS. However, in the hypothalamus, COX-2 mRNA was upregulated only 3 days after treatment. In the amygdala, BDNF and COX-2 mRNAs were downregulated. CRP and corticosterone expression increased with DSS treatment at day 7.

Conclusion: IBD could lead to neuroinflammation in a bottom-up manner, and this effect varied according to brain region. Stress-related hormones and serum inflammatory markers, such as CRP, were upregulated from the third day of DSS treatment. Therefore, early and active intervention is required to prevent psychological and behavioral changes caused by IBD, and region-specific studies can help understand the precise mechanisms by which IBD affects the brain.
\end{abstract}

KEY WORDS: Inflammatory bowel disease; Dextran sodium sulfate; Neuroinfalmmation; Cyclooxygenase 2; Glial fibrillary acidic protein; Brain-derived neurotrophic factor.

\section{INTRODUCTION}

Inflammatory bowel disease (IBD), consisting of Crohn's disease and ulcerative colitis, is a disease causing gastrointestinal tract symptoms such as abdominal pain, vomiting, diarrhea, weight loss, anemia, and anal bleeding. ${ }^{1)}$ Millions of people in the US and Europe experience IBD, and the prevalence of IBD is increasing in southern Europe, Asia, and developing countries, which previously

Received: November 23, 2017 / Revised: January 11, 2018

Accepted: January 12, 2018

Address for correspondence: Jungmin Woo, MD, PhD

Department of Psychiatry, School of Medicine, Kyungpook

National University, 680 Gukchaebosang-ro, Jung-gu, Daegu

41944, Korea

Tel: +82-53-200-5747, Fax: +82-53-426-5361

E-mail:woojm3@hanmail.net

ORCID: https://orcid.org/0000-0001-7180-9972 had a low incidence. ${ }^{2)}$ As approximately $30 \%$ of IBD patients develop symptoms before the age of 21 years, the illness permanently affects the quality of life of the patient. $^{3-5)}$ Even though IBD is a gastrointestinal disease, several studies have shown the presence of neuropsychiatric manifestations, such as depression and cognitive dysfunction. ${ }^{6-8)}$ The prevalence of depressive symptoms is higher in the active phase of IBD. ${ }^{9)}$

The exact mechanism by which depression or cognitive dysfunction occur in patients with IBD, is unknown. However, recent animal studies on the brain-gut axis have provided a direction for understanding the abovementioned neuropsychiatric conditions. These studies revealed that a harmful environment in the gut leads to behavioral deviation, representing depressive and anxiety symptoms, in a bottom-up manner. ${ }^{10-14)}$ The authors sug-

(ㄷ) This is an Open-Access article distributed under the terms of the Creative Commons Attribution Non-Commercial License (http://creativecommons.org/licenses/by-nc/4.0) which permits unrestricted non-commercial use, distribution, and reproduction in any medium, provided the original work is properly cited. 
gest that activation of the hypothalamic-pituitary-adrenal (HPA) axis and immune system, caused by the deviation of the intestinal environment, leads to alteration of the central nervous system. Indeed, previous studies show glucocorticoids, which are increased by activation of the HPA axis, can damage the hippocampus and inhibit neurogenesis. ${ }^{15-18)}$ Glucocorticoids are also involved in the immune system and can trigger the inflammation process. $^{19)}$

Although previous studies in animal models of colitis have addressed stress-related changes in hormones, such as corticosterone, and various gene expression patterns in the brain, there are still some unexplored avenues. ${ }^{20,21)}$ For instance, it is necessary to observe changes in HPA axis-related hormones other than corticosterone, as well as histopathological changes in the brain. It is also important to observe effects in the early stage, i.e. 3 days after dextran sodium sulfate (DSS) treatment, because acute stress also affects the brain and body. ${ }^{22,23)}$

Recent studies on brain-gut interactions have revealed changes brain function initiated in a bottom-up manner by gut inflammation. These changes in the central nervous system are expected to be due to HPA axis activation, changes in the immune system, and the autonomic nervous system, caused by alterations in the intestinal environment, which lead to neuropsychiatric dysfunction and behavioral deviation. ${ }^{24,25)}$ However, in an animal model of colitis, it is necessary to analyze inflammation-related markers in the brain. In this study, we investigated whether chemically-induced colitis could cause changes in the HPA axis response and in the brain at both 3 days, the early stage, and 7 days DSS treatment.

DSS is a chemical that induces IBD-like colitis in animals. To cross the gut-blood barrier and the blood-brain barrier by transmembrane diffusion, a molecule needs to have a high degree of lipid solubility and low molecular weight. DSS is a large, negatively charged molecule (molecular weight 36 to $50 \mathrm{kDa}$ ), and thus does not circulate on a systemic level or reach the central nervous system due to its molecular characteristics. Thus, we can exclude any potential systemic effects and focus on colitis itself. We designed this study to identify the expression of inflammatory markers, glial fibrillary acidic protein (GFAP), and cyclooxygenase 2 (COX-2), as well as brain-derived neurotrophic factor (BDNF) in the hippocampus, hypothalamus, and amygdala. We also observed changes in levels of cortisol, corticosterone, and C-reactive protein (CRP) in the serum, confirming systemic inflammation and changes in the HPA axis.

\section{METHODS}

\section{Samples}

Frozen serum samples and brain paraffin blocks from IBD model mice were received from Laboratory Animal Resources Bank (LAREB, Daegu, Korea). According to information from LAREB, experiments were conducted with C57BL/6J mice (male, 7-8 week). They were purchased from Jung-Ang Laboratory Animal Company (Seoul, Korea). All animals were housed with the maintained in temperature, humidity and light controlled animal rooms $\left(24 \pm 2^{\circ} \mathrm{C}, 50 \pm 20 \%\right.$ relative humidity, $12 / 12$ hour light/ dark cycle with lights on at 07:00) and had ad-libitum access to food and water. To induce colitis for IBD mice model, the mice were treated with $3 \%(\mathrm{wt} / \mathrm{vol})$ DSS (MP Biomedicals, Santa Ana, CA, USA) in drinking water for 3 days and 7 days. The animals were sacrificed at 3 days and 7 days, the blood collection was conducted from abdominal vein with 32-gauge syringe at morning time (10:00-11:00). To minimize pain and stress, the mice were anesthetized with 3\% isoflurane (Hana Pharm., Seoul, Korea). All methods were reviewed and approved by the Animal Care and Use Committee at Pusan National University (IACUC No. PNUH 2016-096). The serum was stored at $-90^{\circ} \mathrm{C}$ for 1 year and brain paraffin blocks were stored at room temperature $\left(21^{\circ} \mathrm{C}-25^{\circ} \mathrm{C}\right)$ for 1 year. Samples were divided into 3 groups $(n=6$ for the control group, $\mathrm{n}=6$ for the 3 day DSS treatment group, and $\mathrm{n}=6$ for the 7 day DSS treatment group).

\section{Serum Analysis}

CRP was measured using a biochemistry analyzer (TBA-120FR; Toshiba Medical System Co., Tochigi, Japan) according to the manufacturer's instructions.

\section{Histopathology}

Tissue sections from the brain paraffin blocks were cut to a thickness of $4 \mu \mathrm{m}$, and hematoxylin and eosin (H\&E) staining was performed using $\mathrm{H} \& \mathrm{E}$ stainer (DAKO CoverStainer; Agilent, Santa Clara, CA, USA). Additionally, tissue sections were stained immunohistochemically using the labelled polymer DAKO EnVision ${ }^{\mathrm{TM}}+$ System-HRP 
(Agilent) according to the manufacturer's instructions. Brain sections were stained with anti-GFAP (ab7260; Abcam, Cambridge, MA, USA) primary antibody. After staining, all areas of the brain that were scanned with the slide scanner (Pannoramic SCAN II; 3DHISTECH Kft., Budapest, Hungary) and images were captured in the hypothalamus, the hippocampus and the amygdala by slide viewer (CaseViewer; 3DHISTECH Kft.). GFAP in hypothalamus, hippocampus, and amygdala was quantified (ImageJ software; NIH, Bethesda, MD, USA) and expressed as a percentage of tissue area, as previously described. $^{26)}$

\section{Enzyme-linked Immunosorbent Assay (ELISA)}

Serum separated from whole blood was used to measure stress levels by ELISA. The concentration of cortisol and corticosterone were determined in a 96-well plate using a cortisol ELISA Kit (FineTest, Wuhan, China) and corticosterone ELISA Kit (Enzo Life Sciences, Ann Arbor, MI, USA), respectively, in accordance with the manufacturer's instructions. Both standard and diluted samples were allowed to react with their antibody and conjugate in a pre-coated plate. Plates were then washed with washing buffer several times. Samples were then were incubated with substrate, and a stop solution was added to the wells. The absorbance was read on a microplate reader at a wavelength of $450 \mathrm{~nm}$ or $405 \mathrm{~nm}$.

\section{RNA Extraction and Real Time Polymerase Chain Reaction (PCR) Analysis}

Total RNA was isolated using an RNeasy Mini Kit and RNase-Free DNase Set (QIAGEN, Hilden, Germany) according to the manufacturer's instructions in each tissue (hypothalamus, hippocampus, and amygdala). The firststrand cDNA synthesis was performed from $1 \mu \mathrm{g}$ of extracted RNA using SuperScript III (Invitrogen, Groningen, Netherlands). To identify expression of the neuroinflam- mation-related marker genes $(B D N F, C O X-2, G F A P)$, real-time PCR from each PCR mixture, containing synthesized cDNA, 2× SYBR Green I Master, 10 pmole of forward-reverse primer, and up to $20 \mu$ of PCR grade water, was performed using the LightCycler 480 system (Roche Diagnostics, Mannheim, Germany). The sequences of the designed primers are shown in Table 1, and Gapdh was used as a control gene. The conditions of the real-time PCR were as follows: pre-incubation at $95^{\circ} \mathrm{C}$ for $5 \mathrm{mi}$ nutes, followed by 35 cycles of amplification at $95^{\circ} \mathrm{C}$ for 10 seconds, $55^{\circ} \mathrm{C}$ for 10 seconds, and $72^{\circ} \mathrm{C}$ for 15 seconds. Data were analyzed with LightCycler 480 Software, and normalization and relative quantification followed the $2^{-\Delta \Delta C t}$ method. The sequences of the primers used are shown in Table 1.

\section{Statistical Analysis}

All values are presented as the mean \pm standard error of mean. Statistical analyses were performed using Student's $t$ test. The value of statistical significance was set at $p<$ 0.05 .

\section{RESULTS}

\section{Effects of DSS-induced Colitis on CRP Levels in Serum and the HPA Axis}

We first measured C-reactive protein (CRP) levels in peripheral blood 3 times to ascertain inflammation induced by DSS in mice. Before treating the mice with DSS, we sampled blood as a control. At 3 days and 7 days DSS treatment, we obtained peripheral blood from the mice to confirm increased CRP level induced by DSS treatment. There was no significant difference in CRP levels between the control group and the 3 days DSS treatment group (Fig. $1 \mathrm{~A}$; control $=0.007 \pm 0.005 \mathrm{mg} / \mathrm{dl}$, DSS 3 days $=$ $0.007 \pm 0.005 \mathrm{mg} / \mathrm{dl}, p=0.500$ ). However, CRP levels of mice increased significantly after 7 days of DSS treatment

Table 1. Primer sequences of the neuroinflammation-related marker genes for real-time PCR

\begin{tabular}{lll}
\hline Gene & \multicolumn{1}{c}{ Forward primer (5'-3') } & Reverse primer (5’-3') \\
\hline BDN & AGCTGAGCGTGTGTGACAGT & GCAGCCTTCCTTGGTGTAAC \\
GFAP -2 & CAGACAACATAAACTGCGCCTT & GATACACCTCTCCACCAATGACC \\
GAPDH & TCTATGAGGAGGAAGTTCGAGA & TGCAAACTTAGACCGATACCA \\
\hline
\end{tabular}

PCR, polymerase chain reaction; BDNF, brain-derived neurotrophic factor; COX-2, cyclooxygenase 2; GFAP, glial fibrillary acidic protein; GAPDH, glyceraldehyde-3-phosphate dehydrogenase. 

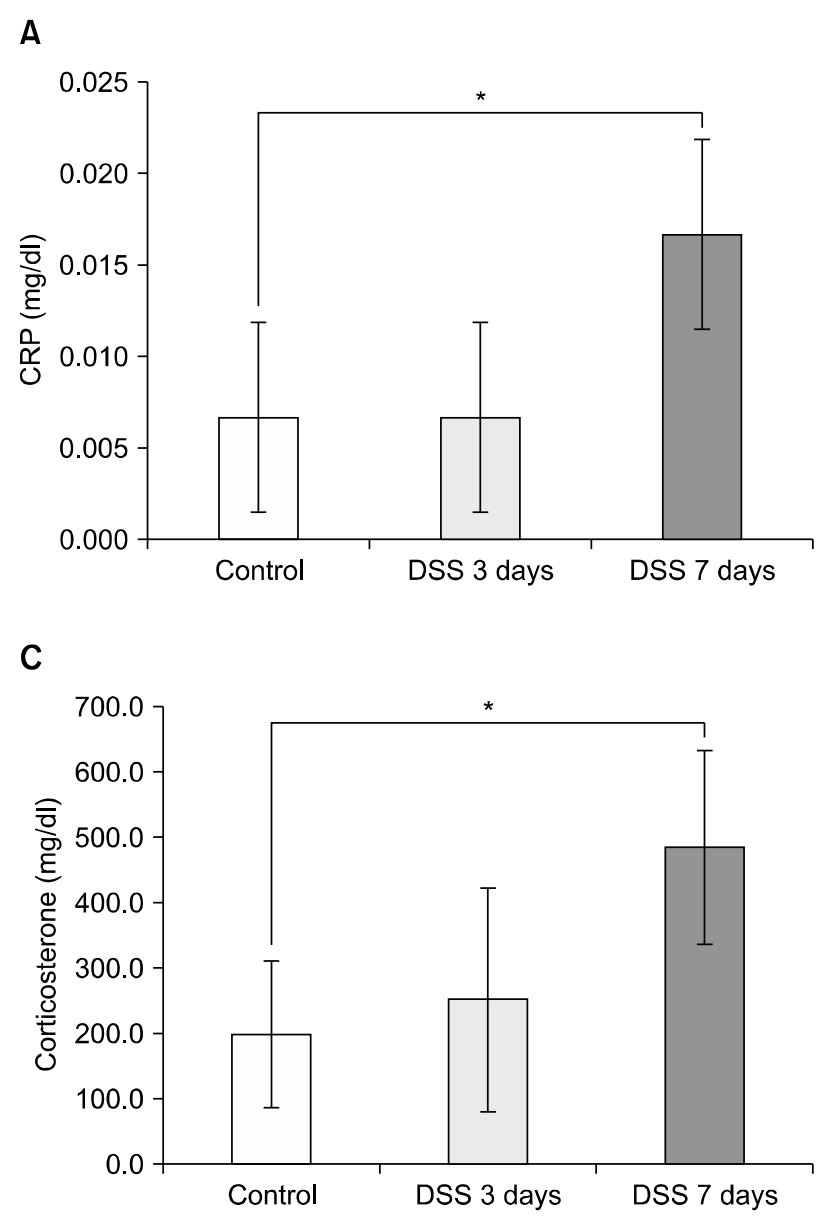

(Fig. 1A; DSS 7 days $=0.017 \pm 0.005 \mathrm{mg} / \mathrm{dl}, p=0.003$ ). Peripheral blood levels of cortisol and corticosterone were then measured to assess the effect of DSS on the HPA axis. Even though cortisol levels in disease induced model mice were increased after dosing them with DSS, it was not statistically significant (Fig. $1 \mathrm{~B}$; control=3.21 \pm 1.16 $\mathrm{ng} / \mathrm{ml}$, DSS 3 days $=3.81 \pm 2.24 \mathrm{ng} / \mathrm{ml}, p=0.570$; DSS 7 days $=5.21 \pm 3.07 \mathrm{ng} / \mathrm{ml}, p=0.180)$. In the case of corticosterone, there was a significant increase after 7 days DSS treatment in mice compared to the control group (Fig. 1C; control $=198.34 \pm 112.10 \mathrm{ng} / \mathrm{ml}$, DSS 3 days $=251.3 \pm 171.83$ $\mathrm{ng} / \mathrm{ml}, p=0.866 ;$ DSS 7 days $=485.20 \pm 148.78 \mathrm{ng} / \mathrm{ml}$, $p=0.013)$.

\section{Effects of DSS-induced Colitis on BDNF mRNA Expression in Different Limbic Structures}

We checked mRNA expression levels of BDNF in the hippocampus, amygdala, and hypothalamus. There was no significant decrease in BDNF mRNA level in the hippocampus at any of the time points tested (Fig. 2A; DSS 3

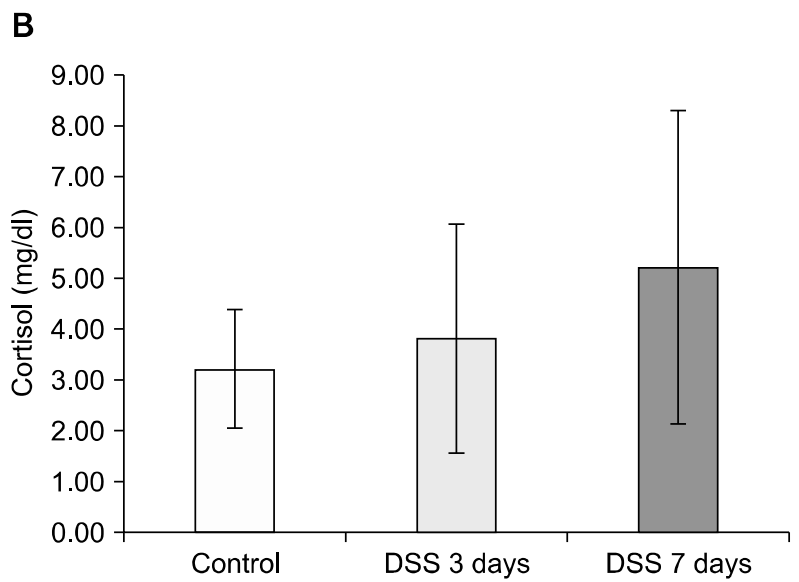

Fig. 1. Chemically induced colitis elevated serum C-reactive protein (CRP) levels and activation of the hypothalamic-pituitary-adrenal (HPA) axis. The graph displays the effect of dextran sodium sulfate (DSS) on serum CRP levels (A), and the effect of DSS treatment on HPA axis (B-C). (B) Cortisol concentration at 0 day, 3 days, and 7 days after DSS treatment. (C) Corticosterone concentration at 0 day, 3 days, and 7 days after DSS treatment. *Significant difference $(p<0.05)$.

days $=0.975 \pm 0.079$, DSS 7 days $=0.970 \pm 0.064$ relative to control), but there was a significant decrease of BDNF mRNA in the amygdala at both 3 and 7 days compared to control (Fig. 2B; DSS 3 days $=0.788 \pm 0.013$ relative to control, $p=0.017$; DSS 7 days $=0.685 \pm 0.041$ relative to control, $p=0.002)$. In the hypothalamus, BDNF mRNA levels were increased significantly at 3 days DSS treatment (Fig. $2 \mathrm{C}$; DSS 3 days $=1.245 \pm 0.088$ relative to control, $p=$ 0.036 ; DSS 7 days $=1.039 \pm 0.059$ relative to control, $p=0.378)$.

\section{Effects of DSS-induced Colitis on COX-2 and GFAP mRNA Expression in Different Limbic Structures}

We then assessed changes in COX-2 mRNA levels in different parts of the limbic system, such as the hippocampus, the amygdala and the hypothalamus of the colitis model mice by relative quantitative PCR to compare relative changes of mRNA expression levels. COX-2 levels in the hippocampus significantly increased after DSS treatment at both 3 and 7 days compared to control (Fig. 3A; 


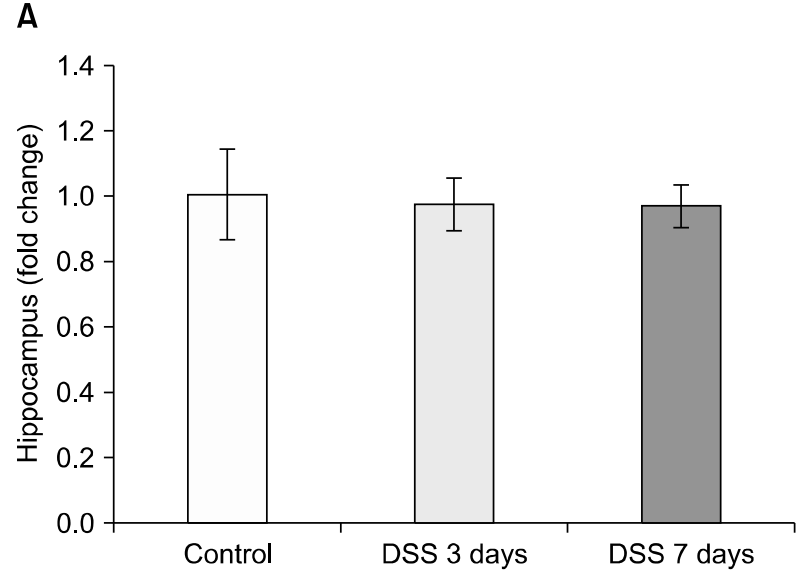

C

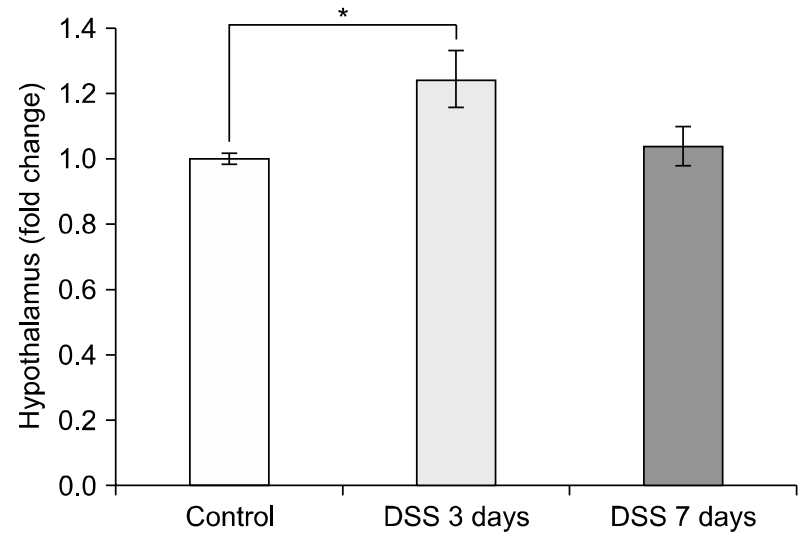

DSS 3 days $=1.273 \pm 0.090$ relative to control, $p=0.018$; DSS 7 days $=1.183 \pm 0.078$ relative to control, $p=0.049$ ). In contrast to the increase in COX-2 levels seen in the hippocampus, COX-2 level in the amygdala decreased compared to controls after dosing mice with DSS (Fig. 3B; DSS 3 days $=0.654 \pm 0.036$ relative to control, $p=0.0007$; DSS 7 days $=0.582 \pm 0.041$ relative to control, $p=0.0008$ ). In the hypothalamus, COX-2 mRNA increased significantly after 3 days DSS treatment (Fig. 3C; DSS 3 days $=3.481 \pm$ 0.579 relative to control, $p=0.017$; DSS 7 days $=0.985 \pm$ 0.102 relative to control, $p=0.825)$. GFAP levels in hippocampus were significantly increased by DSS-induced colitis at both 3 and 7 days after DSS treatment (Fig. 4A; DSS 3 days $=1.351 \pm 0.046$ relative to control, $p=0.024$, DSS 7 days $=1.198 \pm 0.033$ relative to control, $p=0.047$ ). However, the group showed no significant change in GFAP levels in the amygdala (Fig. 4B; DSS 3 days $=0.989 \pm 0.032$ relative to control; DSS 7 days $=1.003 \pm 0.028$ relative to control) and hypothalamus (Fig. 4C; DSS 3 days $=1.085 \pm 0.063$ relative to control, DSS 7 days $=1.007 \pm 0.030$ relative to con-

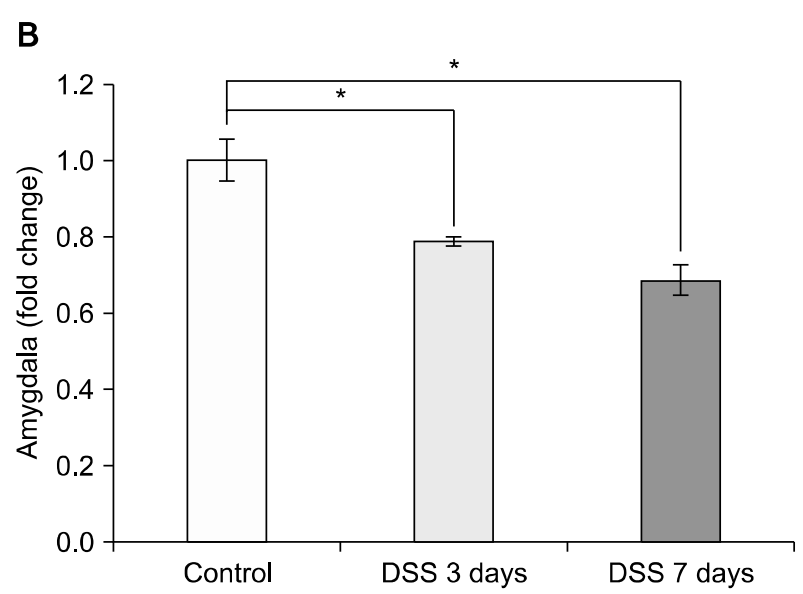

Fig. 2. Dextran sodium sulfate (DSS)-induced colitis led to brainderived neurotrophic factor (BDNF) mRNA changes in different brain regions. Graphs show the effects of DSS-induced colitis in mice on BDNF mRNA levels (A) in the hippocampus, (B) in the amygdala, and (C) in the hypothalamus after exposure to 3 and 7 days of DSS. The values are expressed as fold changes normalized to the untreated control group. The values represent mean \pm standard error of mean; ${ }^{*} p<0.05$.

trol).

\section{Pathological Analysis of GFAP Changes in the Hippocampus, Amygdala, and Hypothalamus in a DSS-induced Colitis Animal Model}

Finally, we confirmed pathological changes with $\mathrm{H} \& \mathrm{E}$ staining and the expression of GFAP in each part of the limbic system using immunohistochemistry (IHC). In histopathological analysis, there were no significant differences in DSS-treated mice at 3 or 7 days by H\&E staining compared with control. To confirm the expression of GFAP, IHC was conducted in different brain regions: the hippocampus, the amygdala, and the hypothalamus. Representative photomicrographs are shown (Fig. 5). GFAP expression in the hippocampus was significantly increased (Fig. 6A; control=8.595 $\pm 0.722 \%$, DSS 3 days= $13.856 \pm 2.639 \%, p=0.032$; DSS 7 days $=12.531 \pm 2.055 \%$, $p=0.033)$. GFAP expression levels in amygdala and hypothalamus were not significant changed during DSS exposure (Fig. 6B: control=1.822 $\pm 0.374 \%$, DSS 3 days $=$ 
A

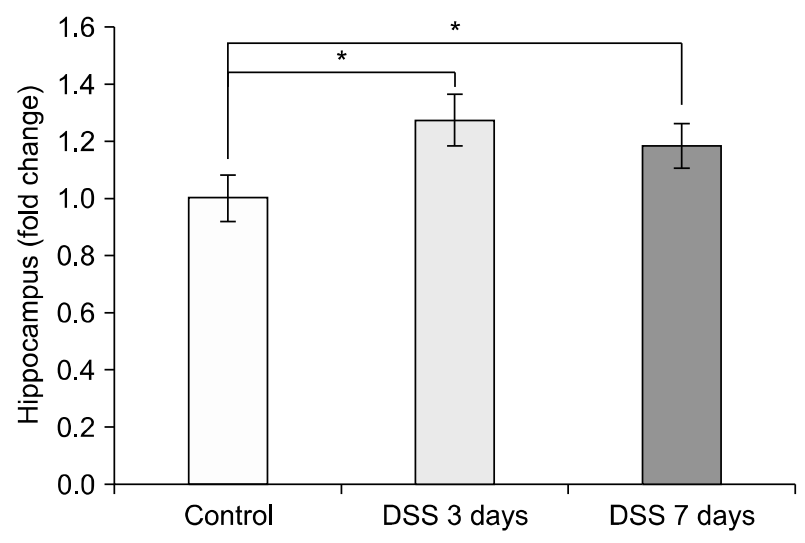

C

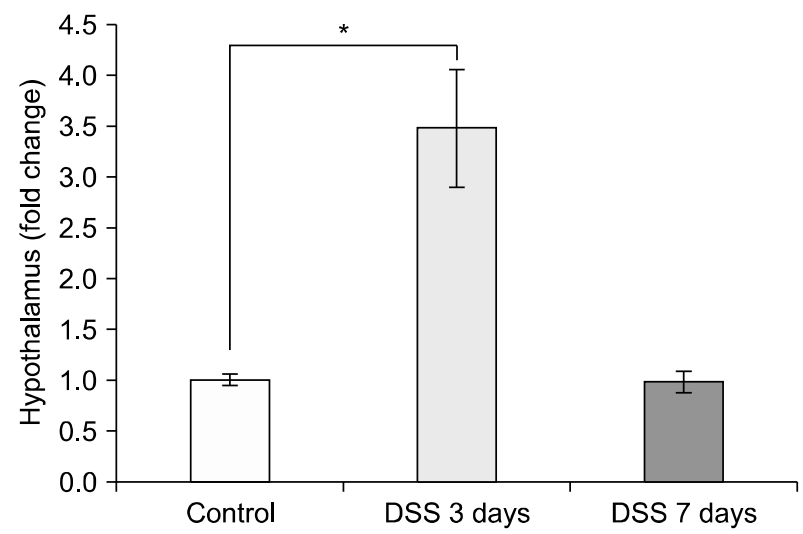

$2.118 \pm 0.777 \%$, DSS 7 days $=3.306 \pm 1.970 \%$; Fig. 6C: control $=2.382 \pm 0.522 \%$, DSS 3 days $=1.999 \pm 0.245 \%$, DSS 7 days $=2.162 \pm 0.606 \%$ ).

\section{DISCUSSION}

The aim of this experiment was to examine whether experimental colitis affects the brain, the changes that occur according to brain region, and how colitis-induced inflammation changes in each brain region. Previous studies have focused on chronic colitis and its effects on the hippocampus ${ }^{27)}$ and gene expression patterns in certain brain regions ${ }^{20)}$; however, these studies are not sufficient in their understanding of the early stage effects of colitis. We attempted to determine whether IBD could affect the central nervous system of IBD disease model mice by identifying changes in mRNA and protein expression associated with inflammation and neuroplasticity in the brain.

In our research, the changes of mRNA expression var-

\section{B}

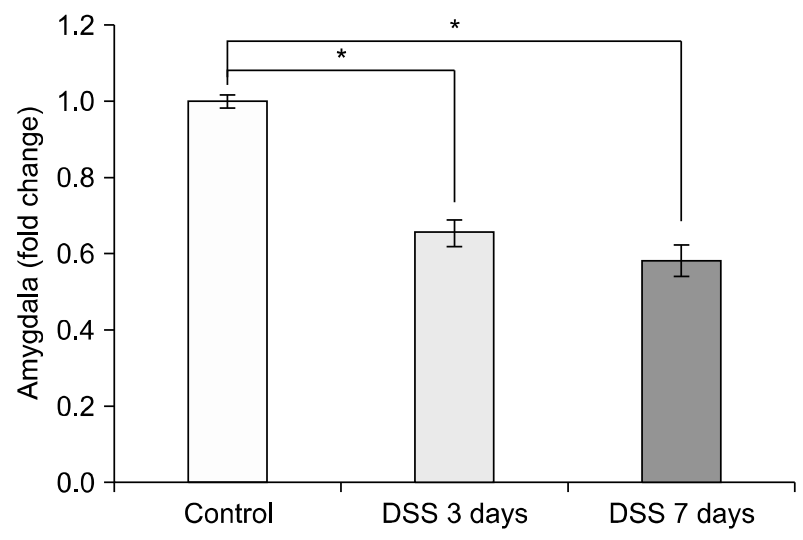

Fig. 3. Dextran sodium sulfate (DSS)-induced colitis caused cyclooxygenase 2 (COX-2) mRNA changes in different brain regions. Graphs show the effects of DSS-induced colitis in mice on COX-2 mRNA levels (A) in the hippocampus, (B) in the amygdala, and (C) in the hypothalamus after exposure to 3 and 7 days of DSS. The values are expressed as fold changes normalized to the untreated control group. The values represent means \pm standard error of mean; ${ }^{*} p<0.05$.

ied depending on the region in the limbic system investigated. At first, COX-2 mRNA, GFAP mRNA, and GFAP expression were upregulated in the hippocampus. COX-2 and GFAP are well-known inflammatory markers in conditions of neuroinflammation and neurodegenerative disease. Cyclooxygenase is a prostaglandin-endoperoxide synthase that forms thromboxane and prostanoids, such as prostaglandins. During inflammatory states in the brain, activated COX-2 can induce production and release of prostaglandin E2 (PGE2). ${ }^{28)}$ In chemically-induced Alzheimer's disease animal model, COX-2 and PGE2 levels in the hippocampus increased simultaneously. This research demonstrated that etoricoxib, a COX-2 inhibitor, could induce recovery from memory impairments, neurodegeneration, and neuroinflammation in the hippocampus, and reduce levels of COX-2 and PGE2. ${ }^{29)}$ GFAP has been also used as marker for reactivity of astrocytes and inflammation in the brain for a long time. ${ }^{30-32)}$ When astrocytes are activated in various neurodegenerative diseases, they increase the expression of GFAP, which is 


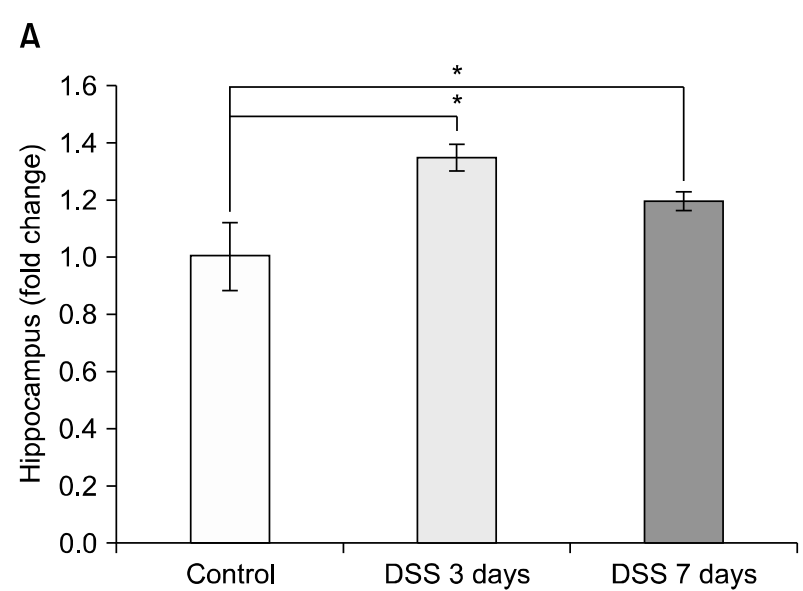

C

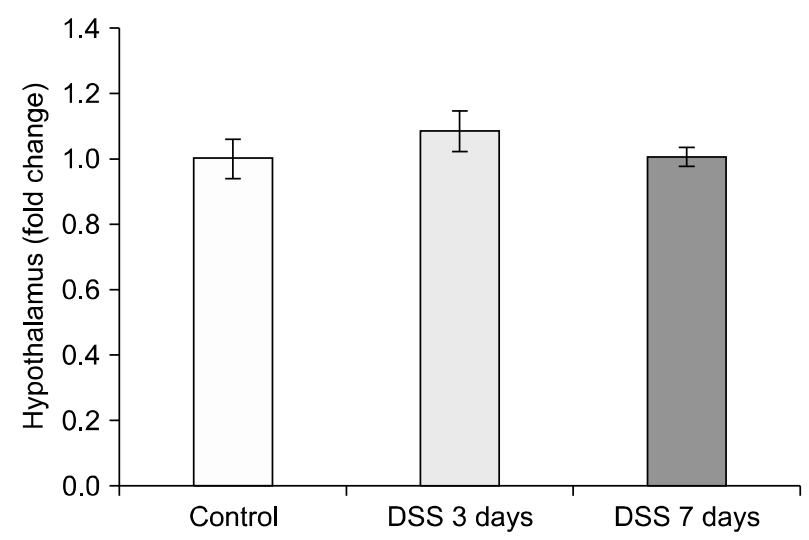

marker for astrogliosis. ${ }^{33-35)}$ In our research, a significant increase of COX-2 mRNA, and GFAP mRNA and protein were observed at early stage colitis, 3-day DSS-treatment, and these changes preceded the significant increase of CRP and glucocorticoids. These results suggest that inflammatory changes in the hippocampus appear early.

In the hypothalamus, BDNF and COX-2 were upregulated 3 days after DSS treatment, but the levels of BDNF and COX- 2 measured 7 days after DSS treatment were reduced to that of the control group. Both the hippocampus and hypothalamus showed elevated COX-2 mRNA level 3 days after DSS treatment, but upregulated COX-2 mRNA expression levels in the hypothalamus had nearly normalized to that of the control group 7 days after DSS treatment. Additionally, there was no increase in GFAP mRNA or GFAP expression after DSS treatment. These results indicate that the hippocampus was more susceptible than the hypothalamus to DDS-induced colitis and the progression rate of hippocampal inflammation is more rapid than that of the hypothalamus, resulting in ex-

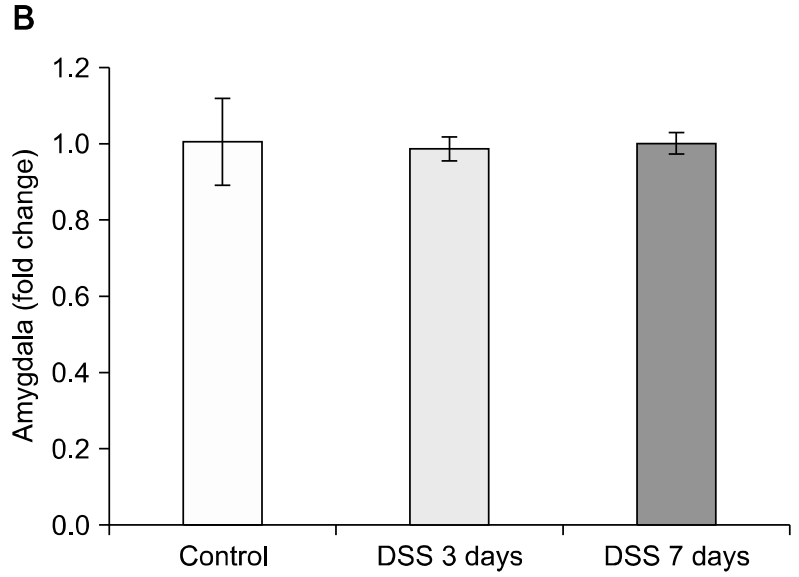

Fig. 4. Dextran sodium sulfate (DSS)-induced colitis lead to glial fibrillary acidic protein (GFAP) mRNA changes in different brain regions. Graphs show the effects of DSS-induced colitis in mice on GFAP mRNA levels (A) in the hippocampus, (B) in the amygdala, and (C) in the hypothalamus after exposure to 3 and 7 days of DSS. The values are expressed as fold changes normalized to the untreated control group. The values represent means \pm standard error of mean; $* p<0.05$.

pression of GFAP before glucocorticoids can sufficiently inhibit expression of GFAP and COX-2. Glucocorticoids have a role in alleviating the progression of inflammation, and several studies have shown that glucocorticoids reduce expression of BDNF, COX-2, and GFAP in the brain. ${ }^{36-41)}$ Thus, elevated activity of the HPA axis affects activation of expression of BDNF and COX-2 in hypothalamus. We also observed that COX-2 and GFAP expression in the 7 day DSS treatment group was decreased in the hippocampus compared to the 3 day DSS treatment group.

The amygdala has important roles in processing sensory input and affective emotion. Even though we evoked systemic inflammation and altered the HPA axis, unlike other limbic structures, significant increases of neuroinflammatory markers in the amygdala, such as COX-2, and GFAP, were not observed. DSS-induced colitis caused downregulation of BDNF mRNA expression and COX-2 mRNA expression in the amygdala. BDNF is a factor related to neurogenesis and neural plasticity. ${ }^{42,43)}$ 


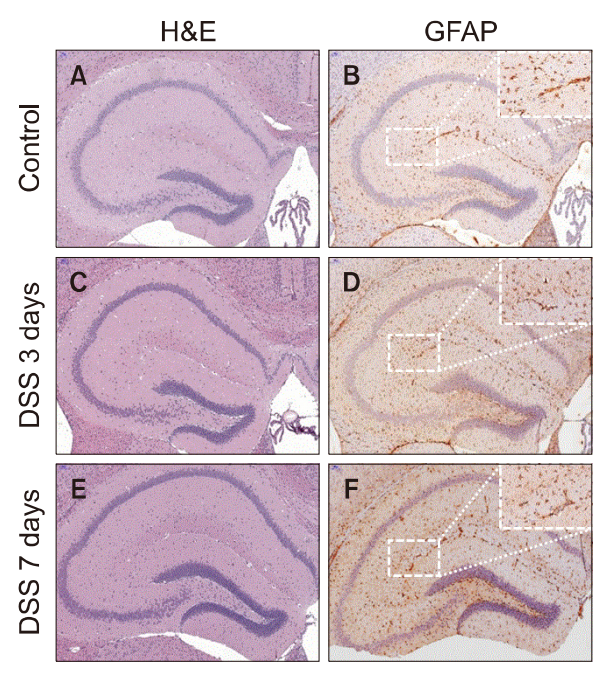

Hippocampus
$H \& E$
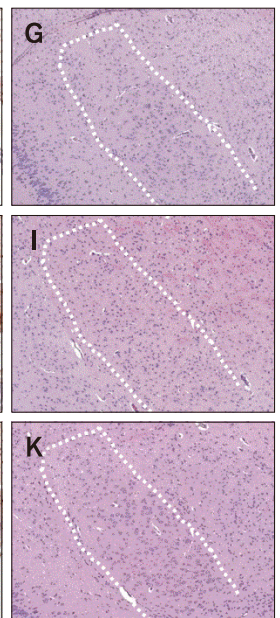

GFAP

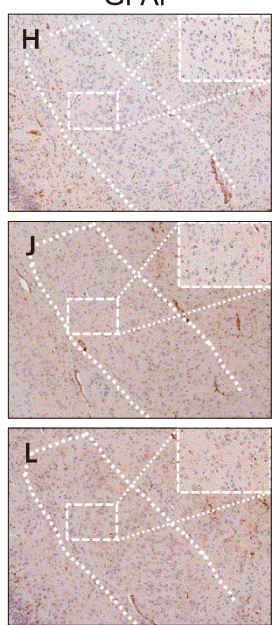

Amygdala
$H \& E$
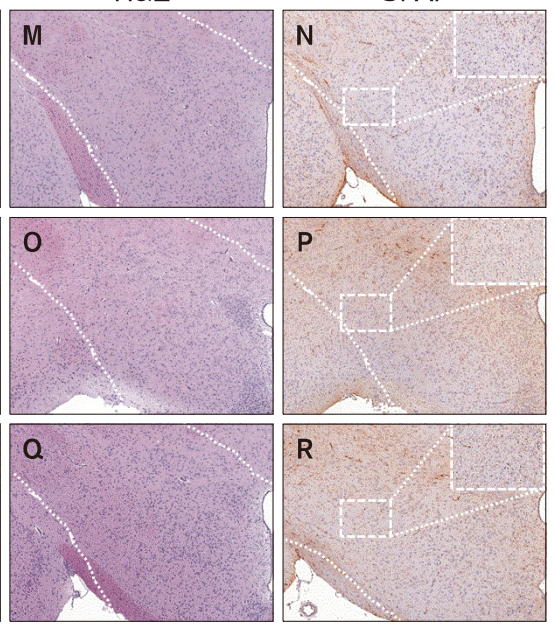

Hypothalamus
GFAP

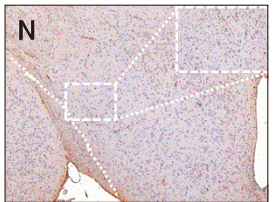

P

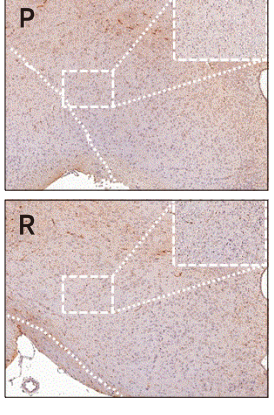

Fig. 5. Glial fibrillary acidic protein (GFAP) expression patterns in the hippocampus, amygdala and hypothalamus after exposure to dextran sodium sulfate (DSS). These are representative photomicrographs of the hippocampus (A-F), the amygdala (G-L) and the hypothalamus (M-R) from our study. Representative histology of the hippocampus was stained with hematoxylin and eosin (H\&E) at untreated (control) (A), 3 days (C), and 7 days (E) after exposed to DSS. GFAP expression in hippocampus tissue was increased compared to (B) control group after exposed to DSS at (D) 3 days, and (F) 7 days, as shown by immunohistochemistry (IHC). Representative histology of the amygdala was also stained with H\&E at (G) untreated (control), (I) 3 days, and $(\mathrm{K}) 7$ days after exposed to DSS. IHC of GFAP expression in the amygdala showed no significant difference between the $(\mathrm{H})$ control group, (J) the 3-day treatment group, and (L) the 7-day treatment group. (M) Hypothalamus tissue stained with H\&E at untreated (control), (O) 3 days, and (Q) 7 days after exposed to DSS. IHC of GFAP expression in the hypothalamus did not show any significant difference between $(\mathrm{N})$ the control group, (P) the 3-day treatment group, and (R) the 7-day treatment group.

BDNF is known to have different expression patterns in response to stress depending on the brain region. Previous studies have shown that the expression pattern of the BDNF gene in the hypothalamus on the 7th day of DSS administration is not different from that of normal mice. ${ }^{21)}$ However, in our results, the expression of BDNF in the hypothalamus after 3 days of DSS treatment was significantly increased. This expression pattern of BDNF can be regarded as a major clue to when the stress period begins.

In stress research, the stress period can be a major factor. Recent studies have reported that neurogenesis of the hippocampus is activated by acute stress. ${ }^{44)}$ In addition, the expression pattern of BDNF responds differently in the brain region depending on the type and severity of stress. When exposed to restraint stress, for 10 days chronically, an increase in BDNF expression is reported in the amygdala region as observed in psychological stress. ${ }^{45)}$ In contrast, no difference or decrease in BDNF expression has been observed in the hippocampus. ${ }^{45)}$ In this experiment, inflammation-mediated stress is thought to differ from that of psychological stress in the brain region.

It has long been proposed that a deficiency of BDNF may lead to psychiatric diseases, especially major depression. ${ }^{46)}$ Systematic review and meta-analysis of the relationship between BDNF protein levels and anxiety disorders show that the BDNF levels of patients with anxiety disorders were lower than those of control groups. ${ }^{47)}$ Postmortem study showed that BDNF levels in the amygdala of women who were diagnosed with major depressive disorder (MDD) was lower than that of the control group. ${ }^{48)}$ In a meta-analysis, unmedicated patients with major depression had a significant decrease in amygdala volume, while the amygdala volume of the antidepressant medicated group was significantly increased. ${ }^{49)}$ Hypometabolism in the amygdala was observed in a patient with treatment-resistant depression. ${ }^{50)}$ In a functional magnetic resonance imaging study, there was a reduced blood oxygen level-dependent signal in the amygdala of patients with ulcerative colitis observed relative to control. ${ }^{51)}$ In summary, it seems that BDNF deficiency in the amygdala is correlated with hypometabolism, volume reduction in depression, and IBD. In contrast to the upregulation of COX-2 in the hippocampus and hypothalamus, there was downregulation of COX-2 in the amygdala. In our study, we have discussed the role of COX-2 in neuro- 
A

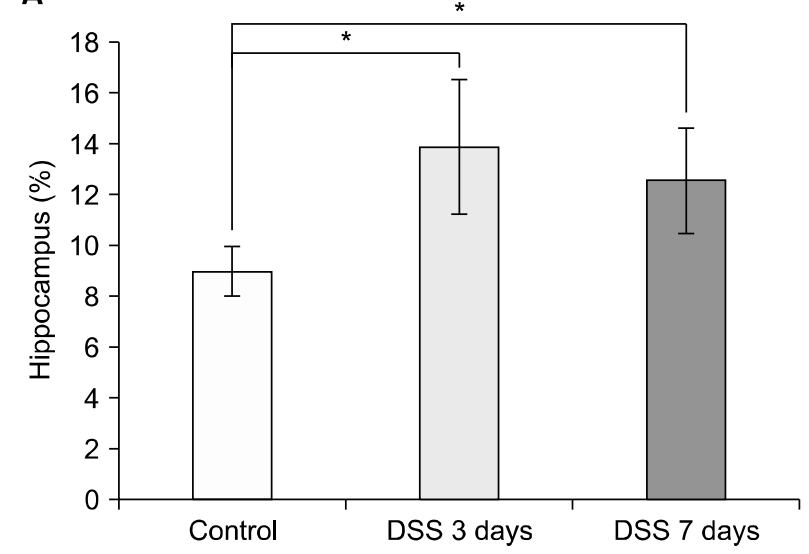

\section{C}

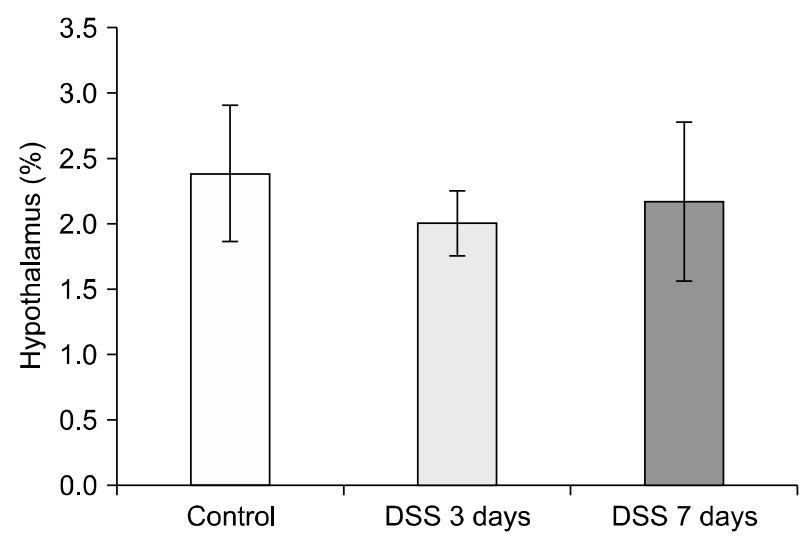

inflammation in the hippocampus and amygdala, but COX-2 also has other roles in brain. Even if inflammation does not occur in the mammalian brain, COX-2 is constitutively expressed in normal physiological conditions, and it has been proposed that COX-2 activity affects synaptic activity and consolidation in the brain. ${ }^{40,52,53)}$ One study found that the activity of COX-2 determines long-term potentiation in the lateral nucleus of the amygdala using a selective COX-2 inhibitor and COX-2 knockout mice. ${ }^{54)}$ Combining our results with previous studies, DSS-colitis affects neural plasticity and neurogenesis in the amygdala by reducing expression of BDNF and COX-2.

Compared with previous studies, our study has yielded some significant findings. Previous studies have focused on whether, and how, external stress affects the HPA axis, and the progression and prognosis of IBD to demonstrate the relationship between psychiatric problems and IBD. It is imperative that we understand how IBD itself affects the
B

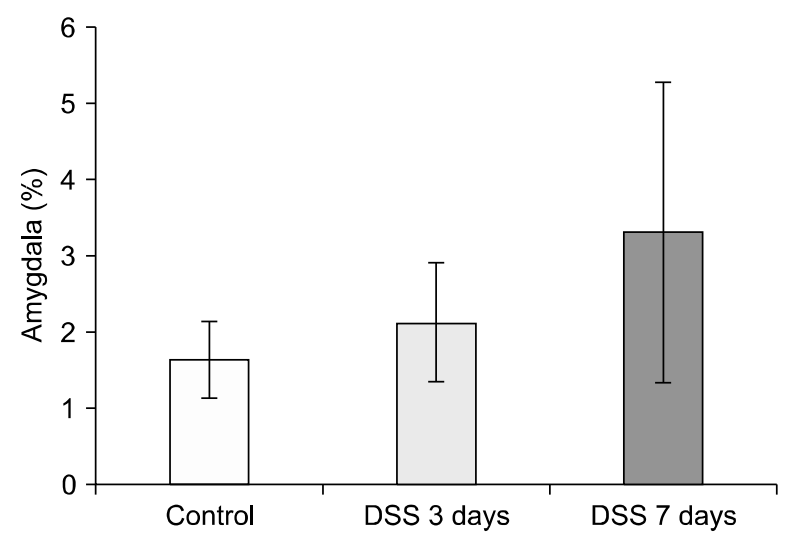

Fig. 6. Quantification of changes in glial fibrillary acidic protein (GFAP) expression in different brain regions. GFAP expression in the hippocampus and amygdala was quantified (Image) software; $\mathrm{NIH}$, Bethesda, MD, USA) and expressed as a percentage of tissue area. (A) GFAP expression changes in hippocampus. (B) GFAP expression changes in amygdala. (C) GFAP expression changes in hypothalamus. Like mRNA expression changes in hippocampus, GFAP expression pattern changed after dextran sodium sulfate exposure. *Significance $(p<0.05)$.

brain to further clarify the bidirectional gut-brain interaction, and the relationship between inflammatory disease and central nervous systems, but there is insufficient research to describe how inflammation in the gut affects the brain. We observed changes in the limbic system at 3 days DSS-treatment, or the early stage, and these changes occurred before glucocorticoids and CRP increased significantly. Unlike in other studies, we harvested samples twice, at 3 days and 7 days after DSS treatment, taking into account the importance of the acute stress response. We found changes in COX-2 mRNA, BDNF $m R N A$, and GFAP mRNA and protein expression patterns 3 days after DDS treatment. Contrary to the claims of the glucocorticoid vulnerability hypothesis, that damage to the hippocampus is caused by overexpressed glucocorticoid in chronic stress, ${ }^{19)}$ this finding suggests that changes in the disease model occur at a very early stage, before changes in the HPA axis, through a pathway independent of glucocorticoid. Therefore, active, early intervention is 
necessary to prevent psychiatric disease because IBD can affect the central nervous systems sooner than we previously expected. Our study also showed that the pattern of inflammation varies depending on the location within the limbic system. A previous study has shown that lipopolysaccharide in the mouse brain can lead to region-specific neuroinflammation. ${ }^{55)}$ In this study, we only present patterns of inflammation in the limbic system in an IBD animal model. In the future, we should examine whether the brain changes in the IBD disease model are the same as those in other inflammatory diseases, or whether these patterns in the brain are universally altered, and how inflammation of peripheral tissue affects the brain bidirectionally.

In addition, histopathological evaluation with $\mathrm{H} \& \mathrm{E}$ staining revealed no significant findings; however, the expression of GFAP started to increase significantly compared to the control group in the hippocampus of DSStreated mice at 3 days, which is representative of the early stage of colitis. Considering this finding, further studies on the early response in IBD-related disease are necessary.

We conclude that the changes in each region of brain caused by DSS-induced colitis were not consistent, and varied depending on location. Unlike inflammation in peripheral tissues, changes in the brain caused by experimental colitis appear to proceed in a planned and orderly fashion, depending on the brain area. Further research is needed to determine whether regional differences are due to differences in susceptibility to systemic inflammation or differences in regional ability to adapt to colitis. Our study suggests that DSS-induced colitis itself could cause neuronal changes in the limbic system at an early stage. It has also been shown that the vulnerability to IBD can vary depending on the region within the limbic system. In this experiment, we did not elucidate the mechanism by which IBD affects the brain. However, as further research on the interaction of the brain with IBD is underway, we hope the mechanism will soon be determined. We believe that early intervention will alleviate the comorbidity of IBD and psychiatric illness and improve the quality of life of patients with IBD.

\section{REFERENCES}

1. Bernstein CN, Fried M, Krabshuis JH, Cohen H, Eliakim R, Fedail S, et al. World Gastroenterology Organization Practice Guidelines for the diagnosis and management of IBD in 2010.
Inflamm Bowel Dis 2010;16:112-124.

2. Loftus EV Jr. Clinical epidemiology of inflammatory bowel disease: Incidence, prevalence, and environmental influences. Gastroenterology 2004;126:1504-1517.

3. Irvine EJ, Feagan B, Rochon J, Archambault A, Fedorak RN, Groll A, et al. Quality of life: a valid and reliable measure of therapeutic efficacy in the treatment of inflammatory bowel disease. Canadian Crohn's Relapse Prevention Trial Study Group. Gastroenterology 1994;106:287-296.

4. Husain A, Triadafilopoulos G. Communicating with patients with inflammatory bowel disease. Inflamm Bowel Dis 2004; 10:444-450; discussion 451.

5. Karwowski CA, Keljo D, Szigethy E. Strategies to improve quality of life in adolescents with inflammatory bowel disease. Inflamm Bowel Dis 2009;15:1755-1764.

6. Bernstein CN, Blanchard JF, Rawsthorne P, Yu N. The prevalence of extraintestinal diseases in inflammatory bowel disease: a population-based study. Am J Gastroenterol 2001;96: 1116-1122.

7. Zois CD, Katsanos KH, Kosmidou M, Tsianos EV. Neurologic manifestations in inflammatory bowel diseases: current knowledge and novel insights. J Crohns Colitis 2010;4:115124.

8. Levine JS, Burakoff R. Extraintestinal manifestations of inflammatory bowel disease. Gastroenterol Hepatol (N Y) 2011;7:235-241.

9. Mikocka-Walus A, Knowles SR, Keefer L, Graff L. Controversies revisited: a systematic review of the comorbidity of depression and anxiety with inflammatory bowel diseases. Inflamm Bowel Dis 2016;22:752-762.

10. Evrensel A, Ceylan ME. Fecal microbiota transplantation and its usage in neuropsychiatric disorders. Clin Psychopharmacol Neurosci 2016;14:231-237.

11. Foster JA, McVey Neufeld KA. Gut-brain axis: how the microbiome influences anxiety and depression. Trends Neurosci 2013;36:305-312.

12. Stilling RM, Dinan TG, Cryan JF. Microbial genes, brain \& behaviour - epigenetic regulation of the gut-brain axis. Genes Brain Behav 2014;13:69-86.

13. Logan AC, Jacka FN, Craig JM, Prescott SL. The microbiome and mental health: looking back, moving forward with lessons from allergic diseases. Clin Psychopharmacol Neurosci 2016;14:131-147.

14. Ghaisas S, Maher J, Kanthasamy A. Gut microbiome in health and disease: Linking the microbiome-gut-brain axis and environmental factors in the pathogenesis of systemic and neurodegenerative diseases. Pharmacol Ther 2016;158:52-62.

15. McEwen BS. Re-examination of the g/ucocorticoid hypothesis of stress and aging. Prog Brain Res 1992;93:365-381; discussion 382-383.

16. Reagan LP, McEwen BS. Controversies surrounding g/ucocorticoid-mediated cell death in the hippocampus. J Chem Neuroanat 1997;13:149-167. 
17. Gould E, Beylin A, Tanapat P, Reeves A, Shors TJ. Learning enhances adult neurogenesis in the hippocampal formation. Nat Neurosci 1999;2:260-265.

18. Sapolsky RM. Glucocorticoids, stress, and their adverse neurological effects: relevance to aging. Exp Gerontol 1999;34: 721-732.

19. Conrad CD. Chronic stress-induced hippocampal vulnerability: the g/ucocorticoid vulnerability hypothesis. Rev Neurosci 2008; 19:395-411.

20. Reber SO. Stress and animal models of inflammatory bowel disease--an update on the role of the hypothalamo-pituitary-adrenal axis. Psychoneuroendocrinology 2012,37:1-19.

21. Reichmann F, Hassan AM, Farzi A, Jain P, Schuligoi R, Holzer P. Dextran sulfate sodium-induced colitis alters stress-associated behaviour and neuropeptide gene expression in the amygdala-hippocampus network of mice. Sci Rep 2015;5. 9970.

22. Bettio LE, Freitas AE, Neis VB, Santos DB, Ribeiro CM, Rosa $\mathrm{PB}$, et al. Guanosine prevents behavioral alterations in the forced swimming test and hippocampal oxidative damage induced by acute restraint stress. Pharmacol Biochem Behav 2014; 127:7-14.

23. Mifsud KR, Saunderson EA, Spiers H, Carter SD, Trollope AF, Mill J, et al. Rapid down-regulation of g/ucocorticoid receptor gene expression in the dentate gyrus after acute stress in vivo: role of DNA methylation and microRNA activity. Neuroendocrinology 2017;104:157-169.

24. Bonaz BL, Bernstein CN. Brain-gut interactions in inflammatory bowel disease. Gastroenterology 2013;144:36-49.

25. Martin-Subero M, Anderson G, Kanchanatawan B, Berk M, Maes M. Comorbidity between depression and inflammatory bowel disease explained by immune-inflammatory, oxidative, and nitrosative stress; tryptophan catabolite; and gutbrain pathways. CNS Spectr 2016;21:184-198.

26. Ruifrok AC, Johnston DA. Quantification of histochemical staining by color deconvolution. Anal Quant Cytol Histol 2001;23:291-299.

27. Zonis S, Pechnick RN, Ljubimov VA, Mahgerefteh $M$, Wawrowsky K, Michelsen KS, et al. Chronic intestinal inflammation alters hippocampal neurogenesis. J Neuroinflammation 2015; 12:65.

28. Font-Nieves M, Sans-Fons MG, Gorina R, Bonfill-Teixidor E, Salas-Pérdomo A, Márquez-Kisinousky L, et al. Induction of COX-2 enzyme and down-regulation of COX-1 expression by lipopolysaccharide (LPS) control prostag/andin E2 production in astrocytes. J Biol Chem 2012;287:6454-6468.

29. Sil S, Ghosh T. Role of cox-2 mediated neuroinflammation on the neurodegeneration and cognitive impairments in colchicine induced rat model of Alzheimer's disease. J Neuroimmunol 2016;291:115-124.

30. Bignami A, Dahl D. Astrocyte-specific protein and neuroglial differentiation. An immunofluorescence study with antibodies to the glial fibrillary acidic protein. J Comp Neurol
1974;153:27-38.

31. O'Callaghan JP, Sriram K. Glial fibrillary acidic protein and related glial proteins as biomarkers of neurotoxicity. Expert Opin Drug Saf 2005;4:433-442.

32. Yang Z, Wang KK. Glial fibrillary acidic protein: from intermediate filament assembly and gliosis to neurobiomarker. Trends Neurosci 2015;38:364-374.

33. Eng LF, Yu AC, Lee YL. Astrocytic response to injury. Prog Brain Res 1992;94:353-365.

34. Eng LF, Ghirnikar RS. GFAP and astrog/iosis. Brain Pathol 1994;4:229-237.

35. Nagele RG, Wegiel J, Venkataraman V, Imaki H, Wang KC, Wegiel J. Contribution of glial cells to the development of amyloid plaques in Alzheimer's disease. Neurobiol Aging 2004;25:663-674.

36. Jacobsen JP, Mørk A. Chronic corticosterone decreases brainderived neurotrophic factor (BDNF) $m R N A$ and protein in the hippocampus, but not in the frontal cortex, of the rat. Brain Res 2006;1110:221-225.

37. Schaaf MJ, De Kloet ER, Vreugdenhil E. Corticosterone effects on BDNF expression in the hippocampus. Implications for memory formation. Stress 2000;3:201-208.

38. O'Banion MK. Cyclooxygenase-2: molecular biology, pharmacology, and neurobiology. Crit Rev Neurobiol 1999;13: 45-82.

39. Schaaf MJ, de Jong J, de Kloet ER, Vreugdenhil E. Downregulation of BDNF $m R N A$ and protein in the rat hippocampus by corticosterone. Brain Res 1998;813:112-120.

40. Yamagata K, Andreasson KI, Kaufmann WE, Barnes CA, Worley PF. Expression of a mitogen-inducible cyclooxygenase in brain neurons: regulation by synaptic activity and g/ucocorticoids. Neuron 1993;11:371-386.

41. O'Callaghan JP, Brinton RE, McEwen BS. Glucocorticoids regulate the concentration of glial fibrillary acidic protein throughout the brain. Brain Res 1989;494:159-161.

42. Lindsay RM, Thoenen $\mathrm{H}$, Barde YA. Placode and neural crest-derived sensory neurons are responsive at early developmental stages to brain-derived neurotrophic factor. Dev Biol 1985;112:319-328.

43. Hofer MM, Barde YA. Brain-derived neurotrophic factor prevents neuronal death in vivo. Nature 1988;331:261-262.

44. Sannino G, Pasqualini L, Ricciardelli E, Montilla P, Soverchia $\mathrm{L}$, Ruggeri $\mathrm{B}$, et al. Acute stress enhances the expression of neuroprotection- and neurogenesis-associated genes in the hippocampus of a mouse restraint model. Oncotarget 2016; 7: 8455-8465.

45. Gray JD, Milner TA, McEwen BS. Dynamic plasticity: the role of glucocorticoids, brain-derived neurotrophic factor and other trophic factors. Neuroscience 2013;239:214-227.

46. Duman RS, Heninger GR, Nestler EJ. A molecular and cellular theory of depression. Arch Gen Psychiatry 1997;54:597-606.

47. Suliman S, Hemmings SM, Seedat S. Brain-derived neurotrophic factor (BDNF) protein levels in anxiety disorders: sys- 
tematic review and meta-regression analysis. Front Integr Neurosci 2013;7:55.

48. Guilloux JP, Douillard-Guilloux G, Kota R, Wang X, Gardier $\mathrm{AM}$, Martinowich $\mathrm{K}$, et al. Molecular evidence for BDNF- and GABA-related dysfunctions in the amygdala of female subjects with major depression. Mol Psychiatry 2012;17:11301142.

49. Hamilton JP, Siemer M, Gotlib IH. Amygdala volume in major depressive disorder: a meta-analysis of magnetic resonance imaging studies. Mol Psychiatry 2008;13:993-1000.

50. Kimbrell TA, Ketter TA, George MS, Little JT, Benson BE, Willis MW, et al. Regional cerebral g/ucose utilization in patients with a range of severities of unipolar depression. Biol Psychiatry 2002;51:237-252.

51. Agostini A, Filippini N, Cevolani D, Agati R, Leoni C, Tambasco R, et al. Brain functional changes in patients with ulcerative colitis: a functional magnetic resonance imaging study on emotional processing. Inflamm Bowel Dis 2011;17: 1769-1777.

52. Breder CD, Dewitt D, Kraig RP. Characterization of inducible cyclooxygenase in rat brain. J Comp Neurol 1995;355:296315.

53. Chen C, Magee JC, Bazan NG. Cyclooxygenase-2 regulates prostaglandin E2 signaling in hippocampal long-term synaptic plasticity. I Neurophysiol 2002;87:2851-2857.

54. Albrecht D. Angiotensin-(1-7)-induced plasticity changes in the lateral amygdala are mediated by COX-2 and NO. Learn Mem 2007;14:177-184.

55. Noh H, Jeon J, Seo H. Systemic injection of LPS induces region-specific neuroinflammation and mitochondrial dysfunction in normal mouse brain. Neurochem Int 2014;69:35-40. 